\title{
Influencia de las cuatro iglesias fundacionales en la trama urbana de Málaga
}

\author{
Influence of the four founding churches \\ in malaga urban pattern
}

\author{
LORENZO MARÍA DíAZ CABIALE \\ Dr. Arquitecto. Profesor Escuela Técnica Superior de Arquitectura de Málaga. Universidad de Málaga \\ lorenzodiaz@uma.es \\ JOSÉ RAMÓN DE ANDRÉS DÍAZ \\ Dr. Ingeniero Industrial. Profesor Titular de la E.T.S.I.I. Universidad de Málaga \\ jrandres@uma.es
}

Recibido: 22/12/2017

Aceptado: $17 / 03 / 2018$

\section{Resumen}

En este artículo se describen las claves del proceso de refundación de la ciudad de Málaga entre 1487 y 1520. Este periodo de la historia del urbanismo de Málaga está enmarcado entre la Málaga Musulmana y la Málaga Conventual. Para entenderlo partimos de una descripción de los parámetros urbanísticos que configuraron la Málaga Musulmana y describimos el proceso de implantación de un nuevo modelo de ciudad contrapuesto al preexistente. El estudio se centra en la influencia que tuvieron las cuatro iglesias intramuros fundadas por los Reyes Católicos en la creación de la nueva trama urbana. Estos templos simbolizaban la nueva realidad político-religiosa y social de la ciudad articulada en cuatro collaciones.

Palabras clave

Málaga, urbanismo, collación, repartimentos, catedral, iglesias. 


\begin{abstract}
The essential component that explain the process involved in Malaga town refounding process is described in this article. This historical period linked to Malaga town planning (1487-1520) is defined between the Muslim and the Conventual Malaga. In order to clarify the issue we place the origin of the analysis related to the urban parameter that shaped the historical Muslim Malaga and so on we describe the establishment of a process that leads to a new town pattern opposed to the previous existent model. The analysis is focused in the influence that the four churches within the town, intramural, founded by the Catholic kings, had in the establishment of the new urban scene. These temples symbolized the new religious, political reality of Malaga town divided in four "collaciones".
\end{abstract}

\title{
Keywords
}

Málaga, town planning, "collación", cathedral, "repartimentos", church.

Referencia normalizada: DÍAZ CABIALE, LORENZO MARÍA - DE ANDRÉS DÍAZ, JOSÉ RAMÓN (2018): “Influencia de las cuatro iglesias fundacionales en la trama urbana de Málaga". Arte y Ciudad. Revista de Investigación, no 13 (abril), págs. 117-142. Madrid. Grupo de Investigación Arte, Arquitectura y Comunicación en la Ciudad Contemporánea, Universidad Complutense de Madrid.

Sumario: 1. Introducción. 2. La ciudad musulmana. 2.1 El espacio doméstico. 2.2. La actividad comercial. 2.3 La función religiosa. 3. Condiciones y características de la reconquista de la ciudad. 4. Los Repartimentos. Espacio privado y espacio público. 5. Málaga a partir de 1487: la iglesia estado. 6. Las cuatros collaciones: los templos y su influencia en la trama urbana. 6.1 La collación de Santiago. 6.2 La collación de San Juan. 6.3 La collación de Los Mártires. 6.4 La collación de Santa María. 7. Conclusiones. 8. Bibliografía.

\section{Introducción.}

El 18 de Agosto de 1487 se produjo la incorporación de la ciudad de Málaga a la Corona de Castilla. Los años posteriores a la toma de la ciudad son definidos por el historiador Enrique del Pino como un periodo de Refundación de la ciudad (Del Pino, 2008: 193).

La brusca y radical transformación sociocultural que experimenta la ciudad se reflejará en un profundo cambio de su estructura urbana. Probablemente, Málaga no volverá a sufrir un proceso similar a lo largo de toda su historia. 
Todos los futuros cambios sociales, políticos, etc., se desarrollarán a partir de ese momento, conservándose una continuidad poblacional, y siempre enmarcados en los parámetros culturales del resto de la civilización occidental.

\section{La ciudad musulmana.}

Málaga se incorporó al dominio árabe desde la llegada del Islam a la Península. Esto significa que en 1487 Málaga es una ciudad con más de 750 años de cultura musulmana. Podemos considerarla, por tanto, como una más de las ciudades musulmanas medievales.

En su obra Málaga Musulmana, F. Guillén Robles (1880) analiza detalladamente la configuración urbana de la ciudad. Adjuntamos copia del Plano de Málaga Musulmana de E. de la Cerda utilizado por F. Guillén Robles para definir su estructura urbana, enmarcada por la muralla límite de la Medina con sus puertas, la Alcazaba, el Castillo de Gibralfaro y los Arrabales.

La morfología urbana de la ciudad quedaba condicionada por su estructura defensiva. Por su situación, puerta del Al-Ándalus y el norte de África, los aspectos militares-defensivos propios del urbanismo musulmán se potencian en el caso de la ciudad de Málaga. El límite de la medina era la muralla. Esta generaba las puertas de la ciudad, que permitía un exhaustivo control del acceso a la medina. La alcazaba y el castillo de Gibralfaro unidos a la costa y entre sí por las corachas constituían, en aquella época, una estructura militar inexpugnable. Este sistema defensivo se completaba con otros elementos como el Castil de Ginoveses y las Atarazanas.

Definida, a grandes rasgos, la estructura militar que la enmarcaba, el análisis urbano de la medina hay que realizarlo en base a los tres grandes usos o funciones de toda ciudad musulmana medieval: la residencial, la actividad mercantil y la función religiosa.

\subsection{El espacio doméstico.}

La religión musulmana supedita la vida de los fieles en todos sus aspectos y manifestaciones. Para analizar la vivienda en la ciudad musulmana Chueca Goitia remarca los versículos 4 y 5 del capítulo XLIX del Corán, denominado "El santuario": "El interior de tu casa es un santuario: los que lo violen llamándote cuando estás en él, faltan el respeto que deben al intérprete del cielo. Deben esperar a que salgas de allí..." (Chueca, 1978: 12). 


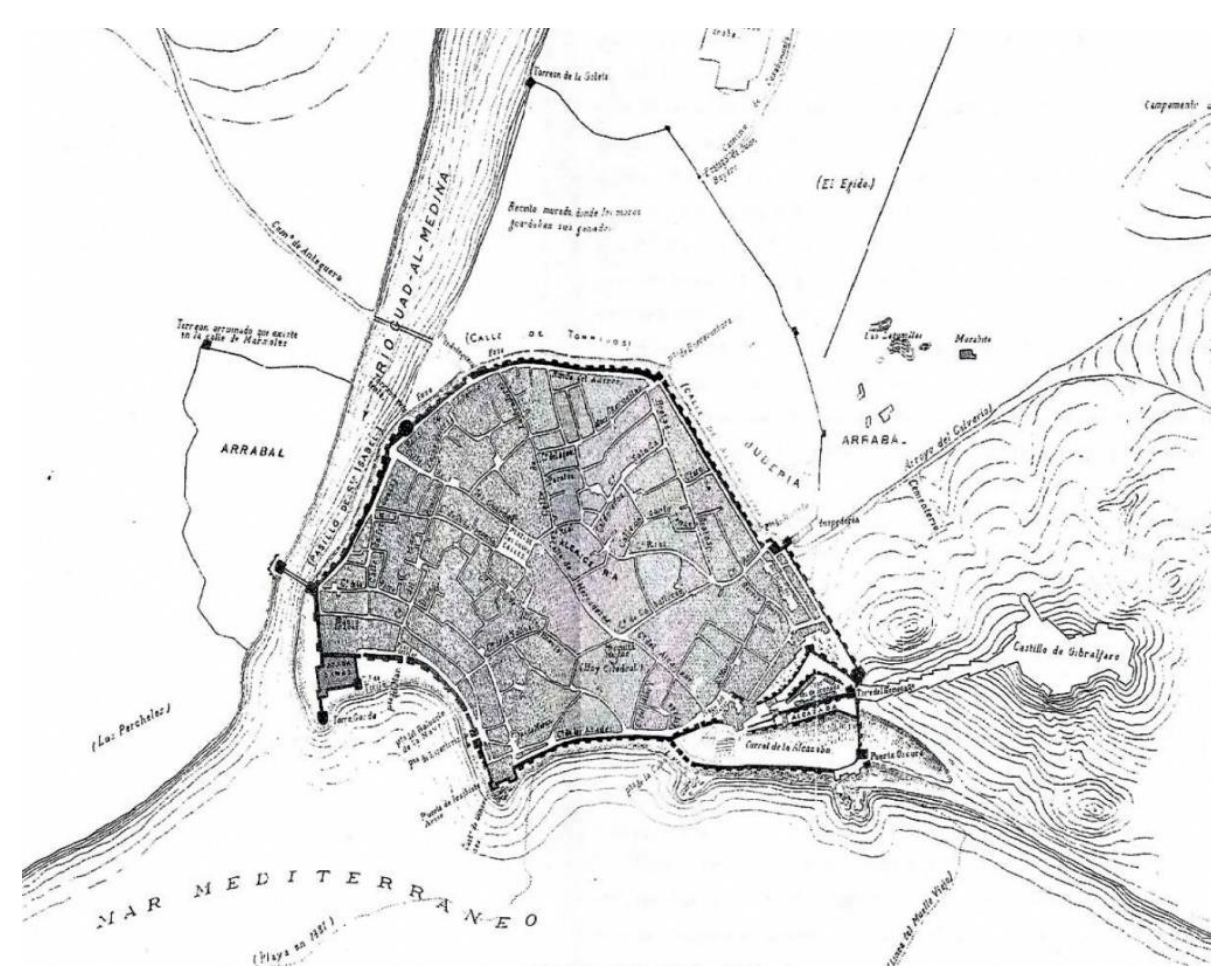

Fig. 1. Plano de Málaga musulmana de E. de la Cerda.

Esta idea de la vivienda como santuario se traduce en una arquitectura absolutamente hermética hacia el exterior. Se produce una reinterpretación de la "casa patio" o del concepto del jardín, creándose esquemas arquitectónicos que doten a la vida doméstica de un espacio al aire libre que no rompa la intimidad buscada.

Este esfuerzo por aislar la vida doméstica también tiene su traducción en los planteamientos urbanísticos de las ciudades musulmanas. Los "barrios" residenciales excluyen cualquier otro tipo de actividad y se convierten en una primera frontera o barrera de protección del espacio doméstico.

En la cultura musulmana la idea del espacio doméstico como santuario llevada a su extremo conduce a la negación del espacio público. “La calle en la ciudad musulmana puede decirse que no existe". En la zona residencial la calle queda reducida al espacio mínimo necesario para poder acceder al "santuario". Cualquier manifestación del "santuario" a un espacio que pueda ser 
usado por alguien ajeno al mismo produciría su desvirtuación o destrucción. El "espacio público" se entiende como un elemento que contamina y daña al espacio doméstico. A partir de aquí se desarrolla todo tipo de mecanismo y estrategias arquitectónicas y urbanísticas para la separación de estos espacios contrapuestos en sus esencias.

Como indica F. Chueca el adarve o calle sin salida podría considerarse el acceso ideal a la vivienda, ya que el tránsito por él queda reducido estrictamente a los usuarios de esas viviendas.

El "misterio" de la ciudad musulmana no deja de ser una celosa búsqueda del espacio doméstico. Las "no calles" niegan cualquier perspectiva o visual que puedan exponer o desnudar al espacio doméstico. El quiebro, el chaflán, la sobriedad y uniformidad de las edificaciones construyen el laberinto idóneo que esconde al "santuario" de las impurezas del exterior.

Con respecto a sus dimensiones, en el caso de Málaga, según los estudios estimativos realizados por Leopoldo Torres Balbas, la población era de 13.000 a 15.000 habitantes (Ruiz Povedano, 2000: 74) y la medina malagueña poseía 2.461 viviendas (Ruiz Povedano, 2000: 89).

Tipologicamente, para analizar la estructura urbana residencial de Málaga L. Machuca analiza las casas encontradas en el barrio de la Alcazaba, las parcelaciones en la "Al medina" de Torrox y el casco viejo de Ronda; estimando las parcelas medias debían estar entre 80 y $100 \mathrm{~m}^{2}$. Las edificaciones tendrían dos alturas como máximo y ocupaciones superiores al 50\% de las parcelas (Machuca, 1997: 136).

\subsection{La actividad comercial.}

En la ciudad musulmana medieval coinciden los espacios de almacenamiento, transformación e intercambio de productos. Estos espacios eran la alcaicería, lo zocos y las alhóndigas.

La alcaicería era el lugar donde se practicaba el comercio de artículos de lujo, en especial de la seda. Partiendo de la vinculación que solía tener este espacio con la actividad religiosa, L. Machuca formula para la medina malagueña una hipótesis de alcaicería encuadrada entre calle Granada y calle Santa Lucía, vinculándola a la Mezquita Aljama o Mezquita Mayor. Esta hipótesis 
la basa en la comparación de la estructura urbana de la medina de Málaga con la ciudad de Fez, considerando ésta el modelo del urbanismo musulmán (Machuca, 1997: 147).

La actividad comercial agrupada en alhóndiga o en los zocos se vuelve a alejar de la idea de calle o espacio público y se vincula más a la idea occidental de edificio. Edificio como lugar donde se desarrolla una función. Podemos decir que la idea de comercio en la ciudad islámica es asimilable a nuestra idea de "mercado" o del "reciente invento" del centro comercial.

De sus alhóndigas la que mejor se ha conocido fue la de Puerta del Mar. Guillén Robles nos menciona otras tres alhóndigas en la medina y José María Ruiz Povedano nos sitúa extramuros los tres zocos "estrictamente citados por las fuentes musulmanas (...): el suq al-haddadin o zoco de los herreros; el suq al Tabbanin o de los mercaderes de la paja y el suq al-gubar, mercado rural del grano o del polvo (...)" (Ruiz Povedano, 2000_92).

\subsection{La función religiosa.}

La influencia de la religión es más directa y patente si cabe en la estructura social y política de la ciudad. El fiel se rige por el Libro Sagrado, esa es su única ley, y solo sirve a su Dios. Cualquier estructura política o de poder supone una interferencia entre el fiel y los designios divinos. Evidentemente existieron todo tipo de estructuras político-territoriales en los territorios musulmanes, pero, a pequeña escala, las ciudades no dejaban de ser una agrupación de fieles regidos por el Corán. En la medina malagueña, al igual que en las otras ciudades nazaríes todo tipo de función pública estaba vinculada a la religiosa a través de la figura del Cadí. Ruiz Povedano nos define y resume la idea islámica del soberano:

como jefe espiritual y temporal de todos los creyentes musulmanes. Éste comprendía y abarcaba unitariamente todas las esferas políticas, administrativas, jurídicas, militares y religiosas, etc., de la comunidad musulmana en la madina de Málaga (...) la representación del poder era ejercida por el Cadí, juez de la comunidad o juez de jueces, por delegación del juez mayor de Granada, que a su vez era nombrado por el sultán (Ruiz Povedano, 2000: 98).

La Mezquita Aljama era el espacio donde el Cadí desarrollaba todas sus funciones además de ser su residencia. En la medina malagueña, al igual que 
en las otras ciudades musulmanas, el urbanismo queda despojado de las funciones y usos vinculados a organismos o instituciones locales tan propias de nuestra cultura. Hoy en día nos resulta difícil reflexionar sobre el urbanismo de cualquier ciudad sin pensar en la influencia de los distintos organismos y administraciones locales. Estos generan edificios singulares y espacios urbanos vinculados a ellos que llegan a vertebrar zonas de la ciudad o incluso terminan convirtiéndose en símbolos de la propia ciudad.

La ciudad musulmana se caracteriza por la ausencia de estos símbolos. Todo se reduce a la religión. El único edificio-espacio-símbolo será la Mezquita Mayor. Málaga poseía una hermosa Mezquita Mayor. Guillen Robles nos reproduce la descripción de 1360 del viajero Iba-Battouta ${ }^{1}$. Nos resulta más revelador para entender la estructura urbana de la medina anterior a $1487 \mathrm{el}$ número y distribución del resto de las mezquitas. Resulta interesantísima la investigación realizada por María Dolores Aguilar (1980: 27-33). A partir de la Cédula de los Reyes Católicos del 23 de Septiembre de 1487 dirigida a sus repartidores confirma la asignación a la Iglesia de "todas las mezquitas que son dentro de los muros de la ciudad" consigue definir las 19 mezquitas que había dentro de la ciudad y una relación de las personas que las tenían a censo a través del Documento de Donación con fecha 9 de Diciembre de 1495 y varios documentos de censos de esas mezquitas que dadas a particulares producían ingresos a la Iglesia.

Analizando los libros de Repartimientos a través de los nombres de los particulares obtenidos consigue localizar el lugar de la mayoría de las mezquitas, identificando ocho de las doce citadas por Guillen Robles. Este estudio fue completado por los trabajos de Må . I. Calero y Virgilio Martínez en su obra Málaga, Ciudad de Al-Andalus. Tras su exhaustivo análisis, María Dolores Aguilar concluye que "es posible que fueran de pequeño tamaño, a veces no ocupan más sitio que una casa y su monumentalidad...sería casi nula...se supone que serían lugares de oración sin la solemnidad de las tradicionales mezquitas...".

Es decir, salvo excepciones como la Mezquita Mayor, para la cultura musulmana no existe la idea de templo. El lugar para orar se aleja de nuestra mentalidad occidental.

${ }^{1}$ (Guillen Robles, 1880: 504) citando a Aben Batuta, Voyages, T. IV pág. 367. 
En el caso de la religión cristiana, el templo, además de un lugar para orar, es un lugar de reunión de los fieles donde se desarrollan los sacramentos. Estos se vinculan al rito, a la liturgia. El templo, ya iglesia, se convierte en un espacio donde existe una función pública. Estos usos convierten a las iglesias en términos funcionales en edificios públicos.

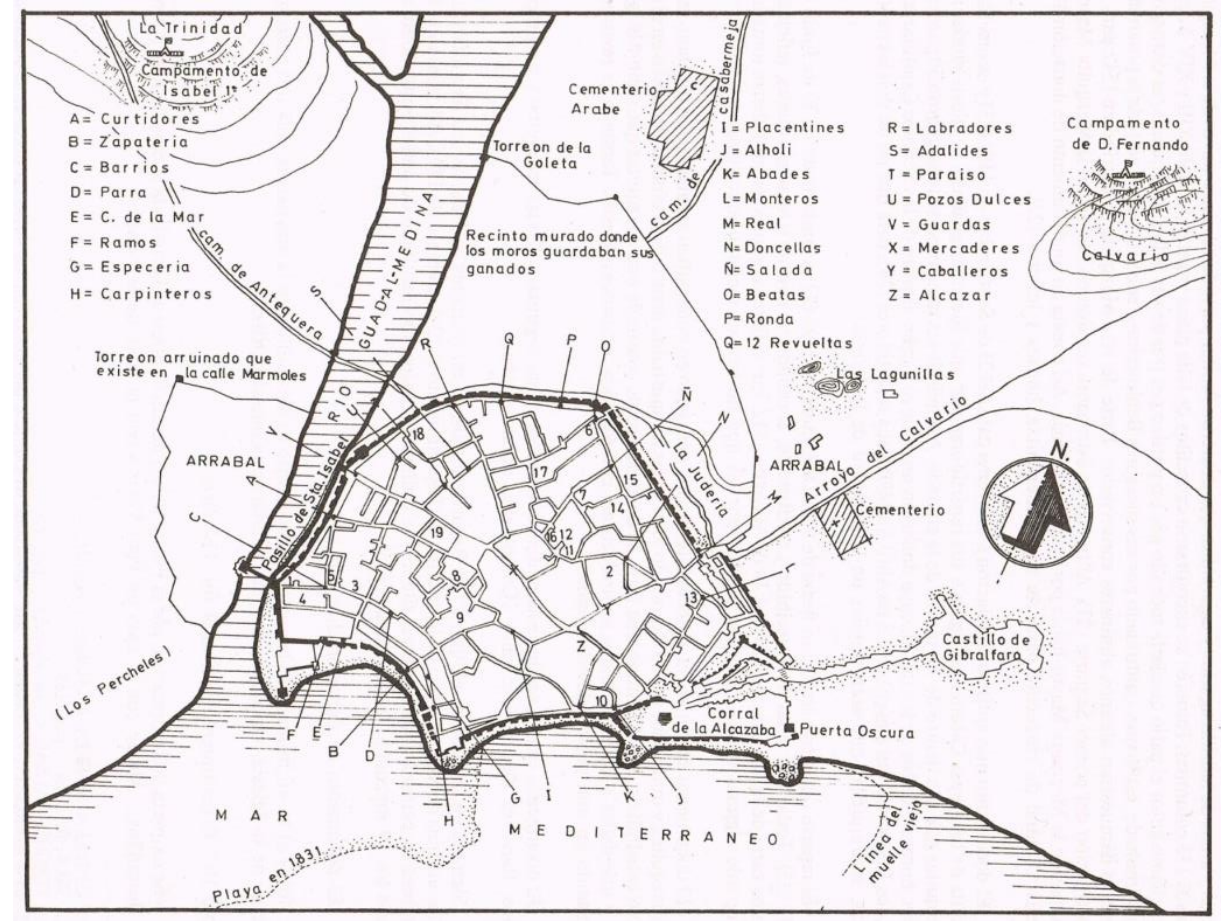

Fig. 2. Localización de mezquitas en el plano de Málaga Musulmana de Guillén Robles (M. D. Aguilar García).

En el caso del Islam no existen todos estos condicionantes. Desvinculado del rito, el templo se reduce al lugar de la oración: el lugar de encuentro del fiel con su Dios. Pero para el Islam, este lugar de encuentro está dentro del propio fiel. En este caso la idea de intimidad es todavía más fuerte que la descrita para el espacio doméstico. En el hogar existe una relación entre personas que exige un espacio físico. En la relación con su Dios la única exigencia física es el propio espacio que ocupa el fiel, que en su postura de alabanza o postración ante su Dios reduce ese espacio al mínimo. Intimidad se identifica con la ausencia de espacio. El espacio no deja de ser una manifestación del mundo externo, un 
lugar externo ajeno al interno. Es probable que la retirada de la alfombra o tapiz por parte del fiel del lugar donde se ha postrado a orar no deje de ser un proceso de destrucción de cualquier "resto" o "memoria" del espacio donde se estableció la relación o comunicación con su Dios. Siendo este mecanismo el máximo exponente de la defensa de su intimidad más profunda. El "templo" o "mezquita" se construiría solo en el momento de la oración y se destruiría al finalizar este acto evitando cualquier tipo de contaminación externa.

\section{Condiciones y características de la reconquista de la ciudad.}

La reconquista de la ciudad se enmarca en la guerra de Granada, una larga etapa de violencia. La rendición de Málaga se produce tras un cruento y demoledor sitio, donde además de los episodios bélicos, el hambre y la peste desolaron la ciudad.

Las capitulaciones del 14 de septiembre de 1478, se realizaron sin concesiones, siendo rechazada con anterioridad por parte del rey la petición de clemencia que hicieron los malagueños, por la resistencia que habían ofrecido. Se especificó en las capitulaciones que la población sin excepción de clase, sexo ni edad quedaría cautiva y confiscados la totalidad de sus bienes: alhajas, casa y tierras. Se fijaron unas detalladas normas para el rescate de los cautivos. Únicamente Ali Dordoux, artífice de las capitulaciones y cuarenta familias más vinculadas a Ali permanecieron en la ciudad como mudéjares. Se les concedió un barrio especial para ellos, dotado de mezquita y baño (Del Pino, 2008: 197). En términos del futuro desarrollo urbanísticos, de las condiciones en que se produjo la reconquista de la ciudad, se desprenden dos factores que marcan su futuro desarrollo: la ciudad reconquistada se encuentra en ruina y se cambia prácticamente la totalidad de la población.

J. M. Ruíz Povedano, escribiendo el desarrollo del cerco de Málaga y su conquista, analiza las acciones llevadas a cabo por la artillería y los bombardeos (lombardas) que sufrió la ciudad. En este sentido cita a Alfonso de Palencia, que se muestra rotundo definiendo, en su obra Guerra de Granada de 1909, las consecuencias de estas acciones bélicas:

En el interior de la ciudad ya no quedaba edificio al que no hubiesen alcanzado los terribles efectos de las balas de piedra disparadas por los morteros desde las primeras horas de la noche hasta el amanecer, con muertes de muchos habitantes". (Ruiz Povedano, 2000: 145, citando a Palencia, 1909: 297). 


\section{Los Repartimientos. Espacio privado y espacio público.}

La deportación de la población musulmana se siguió de la repoblación de la ciudad por parte de los vencedores. En los cuatro últimos meses de 1487 se asentaron en Málaga 1395 personal, casi el 75\% de su futura población. La ciudad se entiende como el botín de guerra a repartir entre los vencedores.

En este sentido el esquema urbano de la ciudad musulmana se presenta como un instrumento válido para el loteo o reparto del botín obtenido.

El detallado análisis de la nueva población, tanto en su procedencia geográfica, como en la nueva configuración de grupos socio-profesionales realizados por Ruiz Povedano nos permite entender la desvinculación total que existe entre la nueva población de la ciudad y el esquema urbano heredado. Esquema basado en unos principios y mentalidad de la cultura musulmana ya descritos totalmente ajenos e incluso rechazados por la nueva población.

El proceso de asentamiento de la nueva población y distribución de los bienes se conoce con el nombre de repartimiento, ejecutado por oficiales reales por encargo expreso de los Reyes Católicos. Se conserva la totalidad de los originales de dicho proceso. Los estudios de los repartimientos realizados por F. Bejarano Robles han culminado con su integro análisis y publicación (Bejarano, 1985, 1990, 1998). Se nombraron repartidores de Málaga a Francisco de Alcaraz y Cristóbal de Mosquera, siendo el primero alcaide de Córdoba.

Este proceso de repartimiento exigió la definición exacta de la ciudad conquistada, mediante su medición y realización de un detallado inventario. Además del recibimiento y asentamiento de los vecinos, y el reparto de casas y bienes entre estos con sus correspondientes cartas de donación (equivalente a un título de propiedad). Se crea por tanto, un registro de bienes y de sus nuevos propietarios.

Este reparto supone uno de los factores más determinantes en el futuro desarrollo urbano de la ciudad. Se abre un periodo de transformación radical de la urbe para adecuarla a los nuevos intereses y necesidades de los nuevos pobladores.

La ciudad se transformará basándose en el concepto de la propiedad privada. Este principio de nuestra cultura occidental lleva a establecer unos límites muy definidos entre el espacio privado y el espacio público. 
Reaparece la idea del espacio público como instrumento de articulación de la ciudad. En el caso de la ciudad de Málaga el proceso de generación del espacio público conlleva la creación de calles y plazas sobre el antiguo tejido de la medina. Este proceso se realizó a través de los repartimientos. En ellos se fijaron condiciones de alineaciones impuestas a los nuevos propietarios, aperturas de calles, creación de plazas, etc...El reparto de propiedades quedó vinculado a una serie de exigencias para garantizar la generación de un nuevo espacio público que estructurase la ciudad. J.M. Ruíz Povedano elabora en su obra un plano de puertas, calles y plazas definiendo las principales redes viarias que articularon la nueva ciudad. En este proceso de creación del espacio público destacan como principales intervenciones intramuros (Fig. 3):

- La apertura de la calle nueva como nuevo eje estructural de la ciudad que une el puerto con el centro de la medina

- La redefinición de la plaza Mayor o Plaza de las Cuatro Calles como espacio público principal de la ciudad.

- La regeneración de la muralla con apertura de nuevas puertas y la creación de un vial paralelo a la misma.

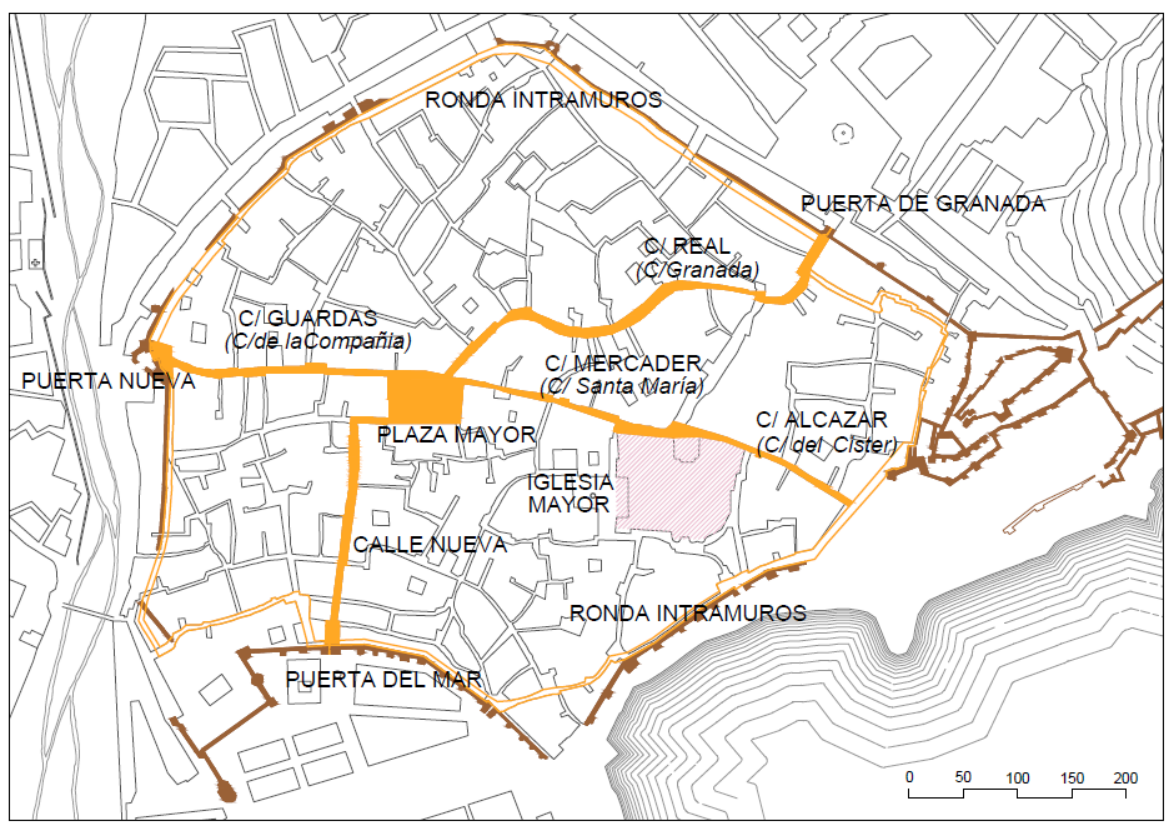

Fig. 3. Esquema resumen realizado sobre copia de la cartografía de Carrión de Mula. 


\section{Málaga a partir de 1487: la iglesia estado.}

Enrique del Pino sintetiza el proceso de refundación de la ciudad en la aplicación del sistema utilizado por los Reyes Católicos para la unificación del territorio: el asentamiento de un doble poder, el político y el eclesiástico.

Para la implantación del primer poder los reyes aplican en la ciudad de Málaga el esquema político de las grandes ciudades castellanas: el cabildo. El corregidor se convierte en la máxima autoridad política con la facultad de nombrar el resto de los cargos. La constitución del cabildo civil fue el 27 de mayo de 1489. El primer corregidor de Málaga fue Garci Fernández Manríquez.

El segundo poder que se implanta en la ciudad marcando su nueva configuración es el eclesiástico. Empleamos el término "poder" intentando remarcar la utilización de la religión por parte de los reyes para la creación y organización de su nuevo reino. La estructura eclesiástica a implantar se realizará a través de la creación de una diócesis regida por el obispo.

Suberbiola Martínez (1975) considera la Iglesia de Málaga la primera Iglesia de estado de España. La ciudad de Málaga sería la primera en aplicar la Bula Ad illam fidei constantiam concedida el 4 de agosto de 1486 por Inocencio VIII a los Reyes Católicos. En dicha bula se les autorizaba a fundar iglesias y a nombrar prebendados y beneficiarios por todo el territorio reconquistado.

El 12 de Febrero de 1488 Pedro González Mendoza, arzobispo de Toledo, confirma el nombramiento de Pedro Díaz de Toledo como obispo de la nueva diócesis de Málaga. Esto se consigue gracias a la voluntad expresa de los reyes Isabel y Fernando de independizar Málaga del obispado de Sevilla. Consiguiendo unificar territorialmente y vincular la nueva diócesis con la nueva implantación política. Pedro Díaz de Toledo era de hecho el capellán y limosnero real. Fue el elegido para estructurar la nueva diócesis y cristianizar los nuevos territorios. Su labor se desarrolló hasta 1949, fecha de su muerte.

Teniendo en cuenta que su sucesor Diego Ramírez de Villaescusa de Haro (1500-1518) acabó centrado en asuntos eclesiásticos que superaban el ámbito de lo malagueño terminando en el obispado de Cuenca; y que los dos obispos siguientes de la diócesis fueron obispos interinos que no la pisaron (Rafael Riario 1518-1520 / Cesar Riario 1520-1525) podemos pensar que la estructura de la diócesis fue definida exclusivamente por Pedro Díaz de Toledo. 
Pedro Díaz tuvo una exquisita formación habiendo cursado en el Colegio de San Bartolomé de Salamanca Teología, Derecho Civil y Canónico. De su nivel intelectual y cultural dan muestras sus traducciones del Fedón de Platón, los Proverbios de Séneca o la Guía de perplejos de Moisés Maimonides. De sus escritos propios destaca Introductio at additionem Josephi super Pentateuchum et alios libros sacrae Scripturae.

Su relación con los personajes más importantes de su época empieza con su nombramiento de capellán del Marqués de Santillana. Fue capellán del rey D. Juan II de Castilla y de Enrique IV. Posteriormente "Secretario del Rey" de los Reyes Católicos. Con su nombramiento de Limosnero Mayor acompañó a la corte en sus desplazamientos en la época de la Guerra de Granada.

Por su cargo organizó la asistencia de heridos en el cerco de Málaga. Este hecho nos puede dar idea de la admiración y autoridad moral que pudo alcanzar entre la futura población de Málaga; culminado de una manera simbólica en su función de portador de la Cruz Profesional en la entrada de los Reyes Católicos de Málaga y su colocación en la parte más alta de la Alcazaba.

El nivel de vinculación que tenía con los Reyes y la estima y confianza que Isabel y Fernando depositaban en él se muestra en las circunstancias de su fallecimiento en Granada el 15 de agosto de 1499 acompañando a los Reyes por solicitud expresa de estos.

Nos hemos extendido en la figura de Pedro Díaz para remarcar el interés de los Reyes en la creación y desarrollo de la diócesis de Málaga únicamente entendible como prueba del modelo territorial del futuro estado cuando se diese por concluida la Reconquista. Muestra de lo cual fue el hecho de ponerla en manos de, probablemente, la persona con más capacidad de gobierno y de mayor confianza de los Reyes durante la época de la Guerra de Granada.

Pedro Díaz será el encargado de materializar en los territorios de Málaga el futuro papel de la Iglesia en el nuevo reino y de diseñar el modelo de ciudad que articularía el futuro reino. Para ello distribuyó el espacio urbano de la Medina original en cuatro parroquias o collaciones: Sagrario, Santiago, Mártires y San Juan. La creación de estas cuatro collaciones no consistía simplemente en situar cuatro edificios sacros en una trama urbana existente y realizar un mero reparto de los futuros fieles, sino establecer una división funcional de una ciudad por reconstruirse y fijar los hitos y símbolos sobre los que se refundarán. 
Podemos afirmar que la estructura parroquial definida para la antigua medina es el hecho más trascendental en el proceso de refundación urbana de la ciudad.

\section{Las cuatros collaciones: los templos y su influencia en la trama urbana.}

La estructura de las collaciones fue tomada de las ciudades castellanas. La delimitación de las parroquias se extrapolaba como un instrumento válido para dividir la población con fines administrativos, militares, etc. En el caso de la ciudad de Málaga esta superposición se llevó al límite llegando a una fusión plena entre la estructura parroquial y las demarcaciones administrativas

Como ya hemos indicado, hay que entender este proceso dentro de su contexto histórico: Málaga se convirtió en el primer ensayo del modelo sociopolítico propuesto por los reyes para articular su futuro reino una vez finalizada la Reconquista.

En el propio proceso de creación de las collaciones se manifiesta este modelo propuesto de iglesia-estado para estructurar el territorio y la ciudad conquistada. Ruiz Povedano relata la singular cronología de los acontecimientos:

Llama la atención que en Málaga este proceso se produjera de un modo distinto e inverso al proceso general. La demarcación administrativa de la ciudad se adelantó a la existencia efectiva de la división territorial eclesiástica de Málaga. Los Reyes Católicos, simultáneamente a la constitución del concejo malagueño, decidieron que en Málaga hubieran cuatro collaciones y que coincidieran sus respectivos ámbitos territoriales con las futuras cuatro iglesias. Sin embargo, por esas mismas fechas, a mediados de 1489 , aún no se encontraban delimitadas las parroquias de la ciudad (...) (Ruiz Povedano, 2000: 377-378).

Esta cronología de los hechos nos muestra que la estructura de las collaciones de la ciudad fue impuesta directamente por los Reyes Católicos y probablemente definida y diseñada por el futuro obispo Pedro Díaz de Toledo

Las collaciones se crearon como una estructura territorial y de articulación de la ciudad donde se unificaba y se consideraba indivisible el poder político y religioso.

Esta estructura de las cuatro collaciones se mantendrá a nivel de parroquias a lo largo de toda la historia de la ciudad hasta nuestros días 


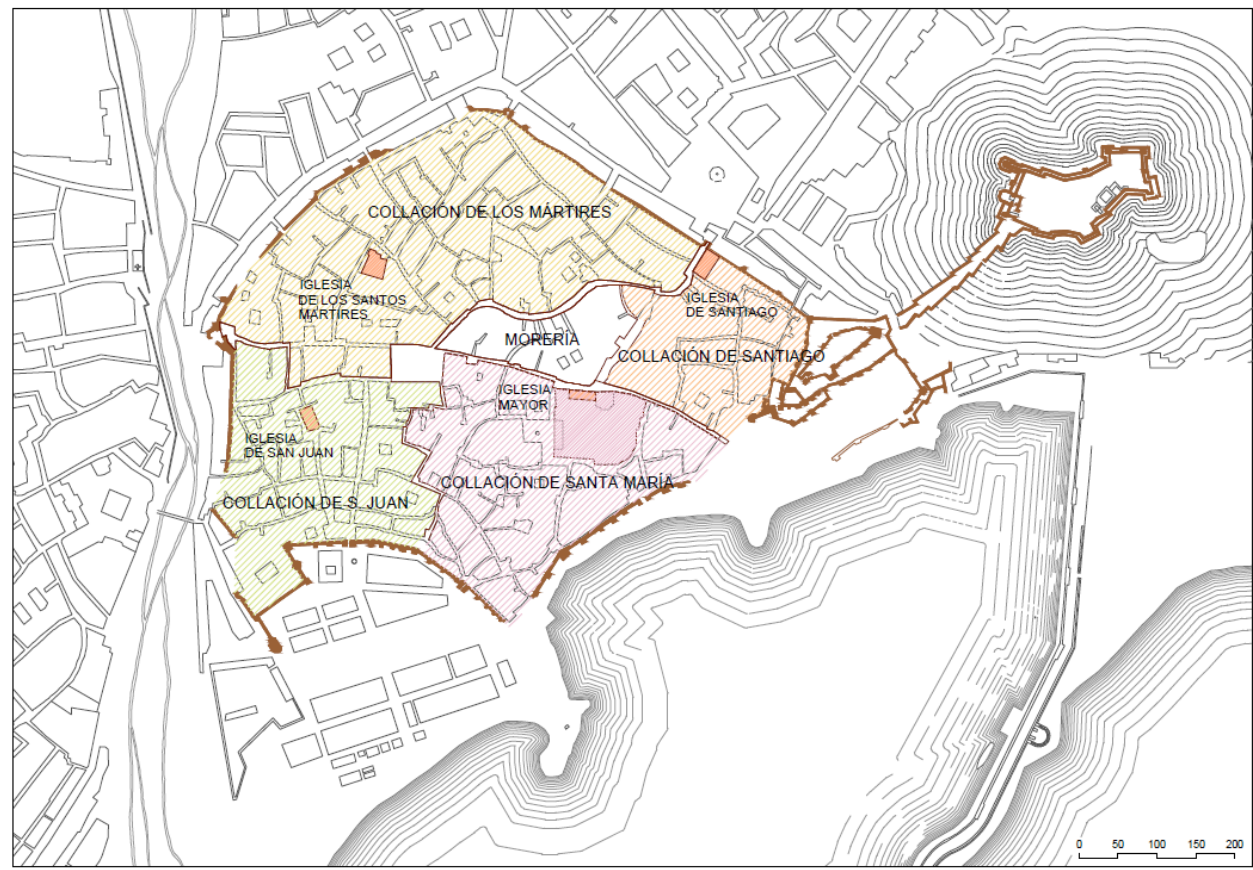

Fig. 4. Plano de las collaciones con la Situación de las Iglesias de Santiago, San Juan, los Santos Mártires y la Iglesia Mayor, realizado sobre copia de la cartografía de Carrión de Mula.

\subsection{La collación de Santiago.}

Los nombres de las collaciones expresan "la mentalidad simbólica del cristianismo medieval" (Ruiz Povedano, 2000: 378, citando a Ladero, 1984: 171172). La advocación de Santiago representa la victoria del cristianismo sobre el Islam. Como nos indica Mónica López en su obra Iglesias de Málaga, cuando los Reyes Católicos tomaban la ciudad se coronaba la torre más alta de su fortaleza con tres estandartes: el de la Santa Cruz, el de Santiago y el Pendón Real. La figura del apóstol Santiago se tomó como protector desde el principio de la reconquista, creándose la leyenda de su aparición al Rey Ramiro I en la batalla de Clavijo donde vence a Abderramán II.

A nivel de población en esta zona residió un elevado número de militares. También se situaron en calle Beatas miembros de la oligarquía malagueña. En la calle Real, convertida en una de las arterias principales de la ciudad, se desarrollaron numerosos comercios (Ruiz Povedano, 2000: 381). 
La iglesia de Santiago, situada junto a la puerta de Granada, potenciaba dicha arteria. Cabe destacar el valor simbólico de su ubicación: El acceso a la ciudad en la toma de los Reyes Católicos. Este hecho queda remarcado en

la resolución por parte del cabildo de que desde Santiago saliese la procesión que conmemoraba la inauguración de la catedral, celebrada el 31 de agosto de 1588, que con gran pompa y boato recorrería la calle Granada hasta la Plaza Mayor y la calle Santa María hasta la puerta de las Cadenas (López Soler, 2011: 88).

Posteriormente se convirtió en el lugar de finalización de la procesión que el 19 de agosto rememoraba el cortejo victorioso de los Reyes.

En el desarrollo urbano de la nueva ciudad, los cuatro edificios de las iglesias de las Collaciones, incluida la Catedral, se erigieron en los símbolos de la nueva realidad urbana. Constituyeron la imagen de la ciudad representando los ideales de su nueva población.

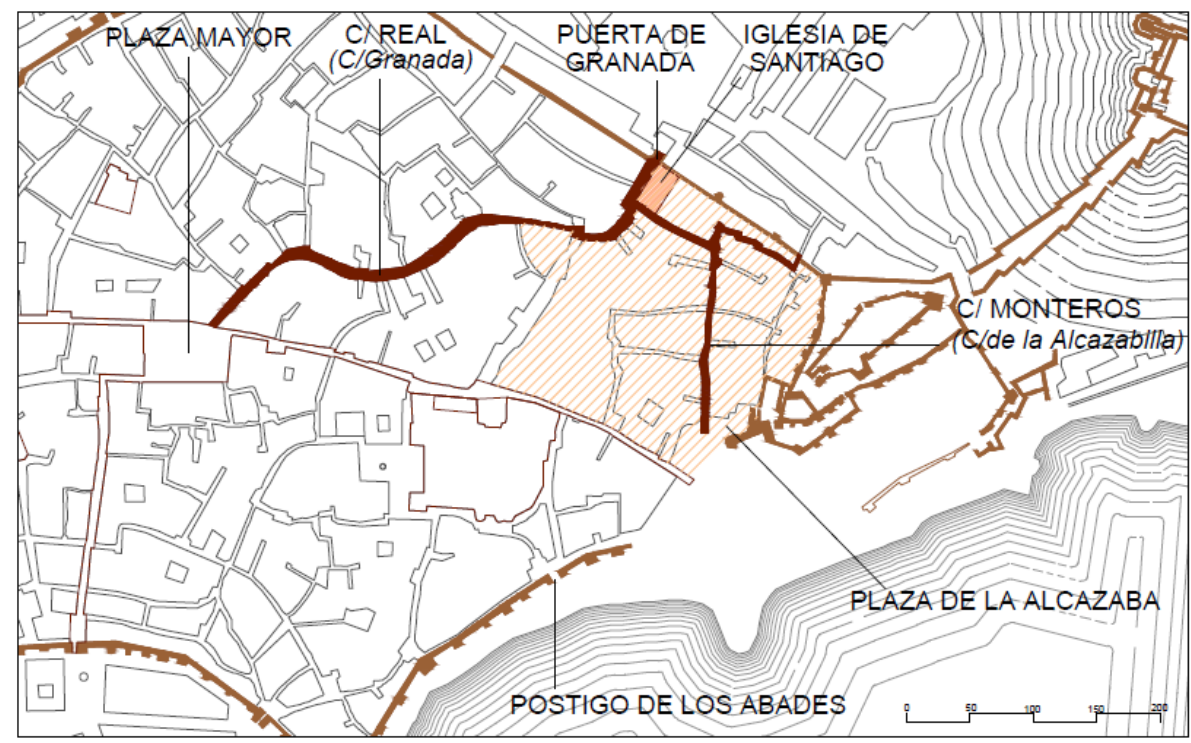

Fig. 5. Esquema gráfico de la estructura urbana de la Collación de Santiago sobre copia de la cartografía de Carrión de Mula.

A nivel funcional se convirtieron en los principales focos de los flujos de la ciudad. En este sentido hay que remarcar que alrededor de cada iglesia se aglutinaron una serie de usos y funciones que potenciaban su entorno próximo y condicionaban el desarrollo urbano de la antigua medina. 
En el caso de Santiago nacieron algunas cofradías de carácter gremial o asistencial, como la congregación del Refugio de Piedad y Amparo de los Pobres o la fundación del Gremio de los Carpinteros (López Soler, 2011: 89).

Desde el siglo XVIII se situó en la esquina de la calle Granada con Tomas de Cozar la cárcel eclesiástica (López Soler, 2011: 89).

Como se aprecia en el esquema adjunto en la estructura urbana de la collación se potencia la unión entre la plaza de la Alcazaba, próxima al postigo de los Abades y la Iglesia de Santiago colindante con la puerta de Granada, redefiniéndose a nivel urbano la calle Monteros (actual calle de la Alcazabilla).

\subsection{La collación de San Juan.}

Actualmente la Iglesia se considera dedicada a San Juan Bautista, pero la mayoría de los autores consideran que su patrono inicial era San Juan Evangelista. En defensa de esta tesis, Mónica López transcribe las reflexiones del jesuita Pedro Morejón, en la segunda mitad del siglo XVII, donde argumenta "entre otras (razones) que en el arco toral de la capilla maior estaba, antiguamente pintada la imagen de San Juan Evangelista" (López Soler, 2011: 183, citando a Morejón, 1999: 236). También refuerza esta teoría la devoción que tenía la reina Isabel a este evangelista.

Fig. 6. Esquema gráfico de la estructura urbana de la Collación de San Juan sobre copia de la cartografía de Carrión de Mula.

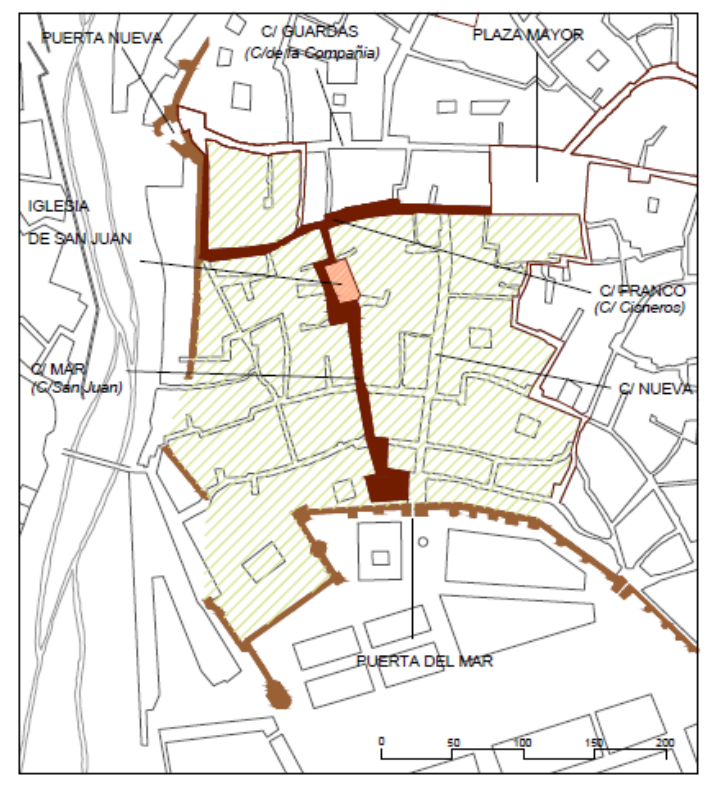


En el año 1726 se realiza una donación a la cofradía del santísimo Sacramento en cuya escritura aparece la advocación de San Juan Bautista. Desde entonces se la designa con este nombre (Camacho, 1981: 198).

La collación de San Juan contenía la principal vía de conexión del puerto con el centro de la ciudad, la Plaza Mayor. Parte de su población tenía actividades relacionadas con la pesca, actividades mercantiles y artesanales.

La situación de la iglesia, centrada físicamente en los términos de la collación condicionó el desarrollo urbano interno de la misma. Adjuntamos esquema en planta donde remarcamos los ejes N-S y E-O que potenció la ubicación del templo. La calle Mar (futura calle San Juan) llegó a rivalizar con la calle Nueva, probablemente potenciada por el tránsito entre Puerta del Mar y la Iglesia. El eje E-O, límite de la collación, generó calle Francos (hoy calle Cisneros) conexión de la Plaza Mayor con la zona Oeste de la muralla. Su proximidad con la Iglesia de San Juan pudo hacer que en importancia alcanzase a la calle Guardas (calle de la Compañía) conexión directa entre la Plaza Mayor y Puerta Nueva.

\subsection{La collación de Los Mártires.}

Aunque en el concejo recibió el nombre de San Sebastián, desde sus inicios cambió su denominación a la de Los Mártires San Cipriano y Santa Paula. Su advocación fue deseo expreso de los Reyes Católicos en cumplimiento de una promesa realizada a los santos durante la conquista de la ciudad. Este hecho queda reflejado en los propios repartimientos (Ruiz Povedano, 2000: 383-384, citando a Guillén Robles, 1874: 508).

La consideración de mártires malagueños, servía para potenciar la idea de considerar el periodo del dominio islámico en la península como un paréntesis histórico producido por la invasión de un pueblo extranjero. Existía, por tanto, un interés en vincular la nueva realidad socio-cultural con el cristianismo de la Málaga romana anterior al periodo musulmán, buscando así una mayor legitimidad de los nuevos pobladores de la urbe.

Entre los equipamientos que surgieron próximos a la iglesia de Los Mártires destaca en 1571 la creación del Hospital de Convalecientes y en 1682 se vuelve a fundar el Hospital de San Julián, llevado por la Hermandad de la Caridad (López Soler, 2011: 133-134). 
La iglesia situada en la zona céntrica de la collación, articula su estructura interna. Potencia el eje de unión con la Puerta de Buenaventura y se vincula a dos de las arterias principales de la nueva ciudad: se une a la calle Real (calle Granada) mediante la calle Labradores (calle Santa Lucía) y se comunica con calle Guardas (calle de la Compañía) mediante la calle Paraíso (calle de los Mártires). La iglesia termina configurando los ejes:

- Muralla Oeste (Puerta de Antequera)- Iglesia de los Mártires - CalleReal (calle Granada) mediante calle Labradores (calle Santa Lucía)

- Puerta Buenaventura - Iglesia de los Mártires - calle Guardas (calle de la Compañía) mediante calle Buenaventura (calle Plaza del Teatro - calle de las Comedias) y calle Paraíso (calle de los Mártires).

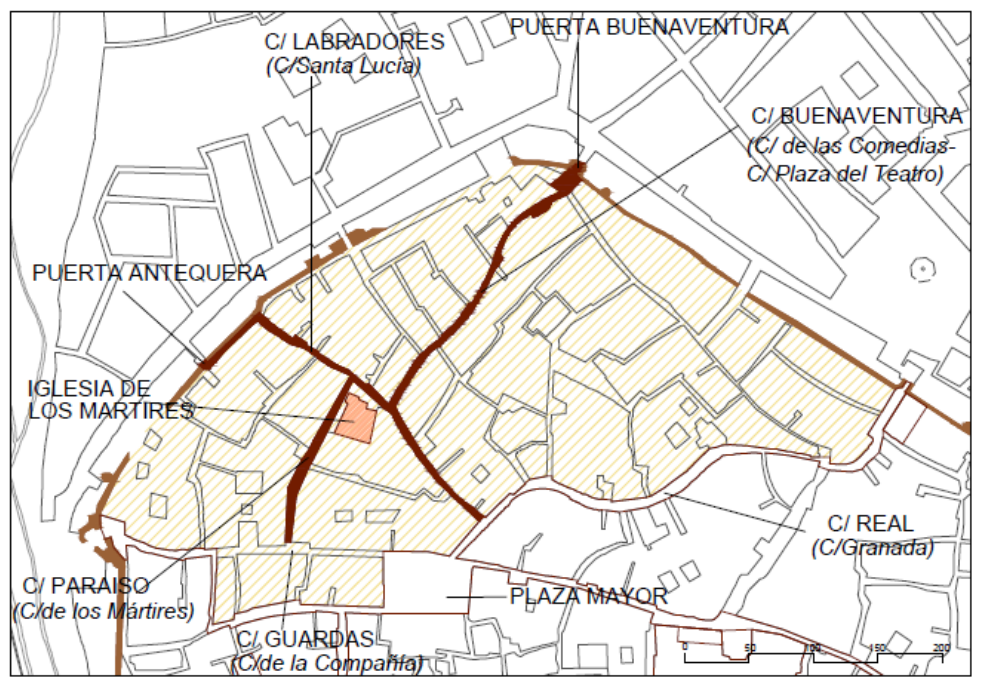

Fig. 7. Esquema gráfico de la estructura urbana de la Collación de los Mártires sobre copia de la cartografía de Carrión de Mula.

\subsection{La collación de Santa María.}

En esta transformación de la Málaga musulmana a la Málaga cristiana, en términos urbanísticos hay que considerar globalmente el conjunto mezquitacatedral. En este periodo, a nivel de configuración urbana, la mezquita aljama transformada al culto cristiano generando la Iglesia Mayor o de Santa María de la Encarnación (Iglesia Vieja, primitiva catedral) forman una unidad que estructura y ordena la collación de Santa María y gran parte del resto de la medina. 
Existen distintas hipótesis sobre la situación exacta de la Mezquita Mayor de la antigua medina, en relación con la catedral, siendo especialmente interesante la elaborada por María Dolores Aguilar (1985: 68-69) y recogida en el estudio sobre la Iglesia del Sagrario, de Rosario Camacho y José María Romero (1987: 3-4). En este estudio también plantean un esquema en planta de la posible Iglesia Vieja.
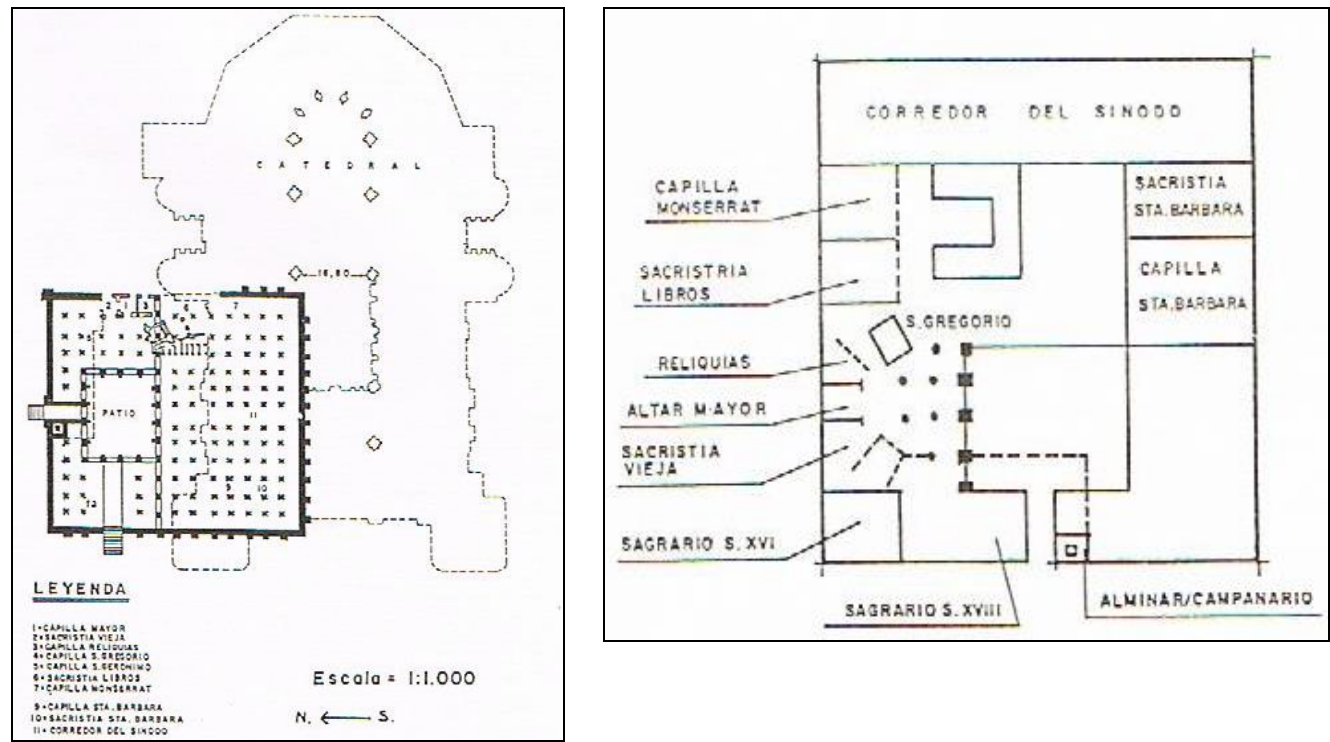

Figs. 8 (izq.). Planta de la mezquita en relación con la catedral (Málaga) y 9 (drch.) Croquis con la distribución de espacios de la Iglesia Vieja. Esquema de situación del Sagrario. M.D. Aguilar García, copia de la lámina 1 de "La mezquita mayor de Málaga y la iglesia vieja".

El conocimiento que tenemos de la mezquita, además de algunos restos arqueológicos $^{2}$ se basa en las descripciones de los viajeros musulmanes como los ya citados de Aben Batuta, que destaca la belleza de su patio (Guillén Robles, 1874: 504). J. M. Ruíz Povedano nos aporta los testimonios de los primeros cristianos que a partir del 18 de Agosto 1487 tuvieron ocasión de conocerla: Pere Llitrá que resalta sus numerosas columnas de mármol y jaspe y que la considera más pequeña que la de Córdoba. La de Gerónimo Münzer que en 1495 describe las 113 columnas exentas (Ruiz Povedano, 2000: 94).

${ }^{2}$ Catálogo de Protección Arqueológica de Málaga. Yacimiento no 70. Restos de la Mezquita Mayor. 
El edificio símbolo de la nueva realidad de Málaga va generando en su proceso de creación las calles colindantes del conjunto y potencia los ejes de conexión con la Plaza Mayor y con la Puerta de Granada. Se redefine por tanto la calle Mercaderes (actual calle Santa María) como arteria principal que vincula la Plaza de las Cuatro Calles con la Iglesia Mayor. Este eje se prolonga redefiniendo la calle Alcázar (calle Cister) hasta la Plaza de la Alcazaba-Aduana.

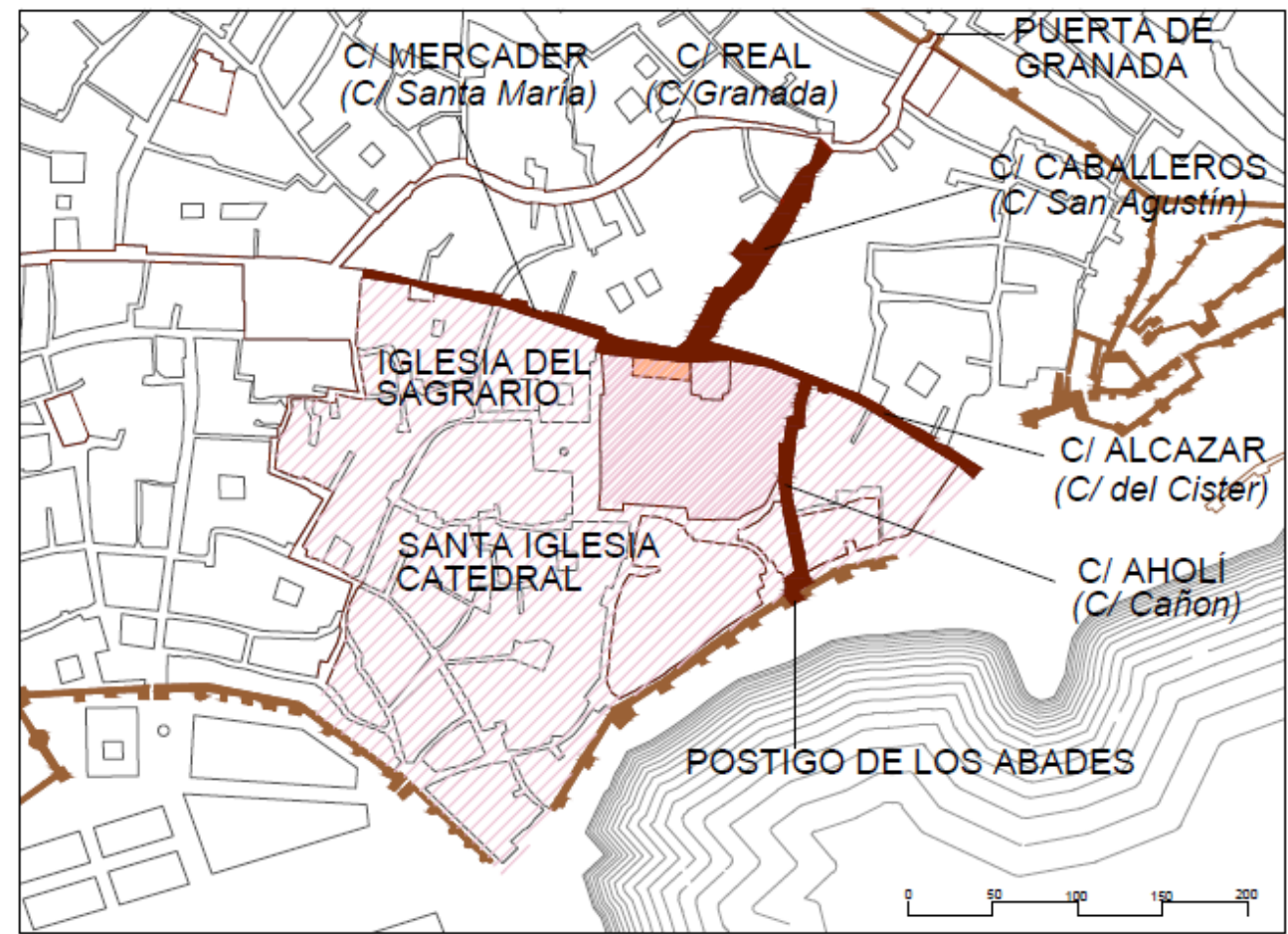

Fig. 10. Esquema gráfico de la estructura urbana de la Collación de Santa María sobre copia de la cartografía de Carrión de Mula.

El otro eje que se potencia es la unión de la Iglesia Mayor con el principal acceso de la ciudad (Puerta de Granada). Estructurando en este caso parte de la collación de Santiago, se genera calle Caballeros (calle San Agustín) enlazando con el último tramo de la Calle Real. Su conexión más directa con la muralla del litoral se realizaba a través de la puerta Postigo de los Abades. Esta conexión se configura a través de los límites del complejo de la Iglesia Mayor generando la calle Alholí (calle Cañón). 


\section{Conclusiones.}

Existe un periodo de la evolución urbana de Málaga, que podríamos denominar la Málaga de las collaciones comprendido entre los años 1487 y 1520, situado por tanto entre la Málaga musulmana y la Málaga conventual.

Durante estos treinta y tres años se produce el proceso de transformación urbana de la Málaga musulmana a la Málaga cristiana. En este periodo, la ciudad se refunda recuperando el concepto de espacio público. Esta nueva idea de ciudad, fruto de la nueva realidad socio política de sus nuevos habitantes, conllevó la apertura de calles y plazas.

Este proceso de transformación se desarrolló reflejando en la trama urbana el nuevo sistema político-administrativo importado de la ciudad castellana. Este sistema unificaba el poder político con el religioso.

La creación de la diócesis de Málaga y la implantación de las cuatro parroquias o collaciones se entienden como hechos impuestos directamente por los Reyes Católicos siendo definidos y ejecutados por el primer obispo de Málaga Pedro Díaz de Toledo.

La estructura de las cuatro collaciones se convierte en el instrumento de ordenación urbana de la nueva ciudad.

Las iglesias o templos de las cuatro collaciones se erigen en los hitos urbanos y en los símbolos de la nueva realidad social y política. Estos hitos, atraerán un gran número de las nuevas funciones desarrolladas en la antigua medina (hospitales, asociaciones gremiales, etc) marcando los nuevos flujos urbanos y condicionando parte de su desarrollo.

Para expresar la idea de ciudad, Fernando Chueca Goitía recurre a las teorías de Spengler afirmando:

lo que distingue la ciudad de la aldea no es la extensión, no es el tamaño, sino la presencia de un alma ciudadana (...) el verdadero milagro es cuando nace el alma de una ciudad. Súbitamente, sobre la espiritualidad general de la cultura, destácase el alma de la ciudad como un alma colectiva de nueva especie, cuyos últimos fundamentos han de permanecer para nosotros en eterno misterio. $\mathrm{Y}$ una vez despierta, se forma un cuerpo visible (Chueca Goitia, 1978: 16-17, recogiendo cita de Spengler, 1946: 131). 
Esta formación del Geist, espíritu o alma de la ciudad se suele cimentar y estructurar en torno a símbolos que aúnan y representan los deseos y aspiraciones de sus habitantes. La arquitectura en sus principales manifestaciones (edificación y urbanismo) suele ser un instrumento ideal para generar estos símbolos. En este sentido, hemos querido mostrar cómo las cuatro parroquias fundacionales forman parte del Geist de la ciudad de Málaga.

\section{Bibliografía.}

Aguilar GarcíA, María Dolores (1973): “La Iglesia de Santiago de Málaga" en Revista Jábega no 2. Centro de Ediciones de la Diputación de Málaga. Málaga.

AgUiLAR GARCíA, María Dolores (1980a): Málaga Mudéjar. Arquitectura religiosa y civil. Universidad de Málaga. Diputación Provincial de Málaga. Málaga.

Aguilar García, María Dolores (1980b): “La armadura de la Iglesia de San Juan. Málaga" en Estudios de Arte, Geografía e Historia, no 3. Universidad de Málaga.

Aguilar García, María Dolores (1985): “La mezquita mayor de Málaga y la iglesia vieja." en Boletín de Arte nํㅜ 6. Universidad de Málaga.

Aguilar GarcíA, María Dolores (1988): “La Plaza Mayor de Málaga siglo XVI." en Boletín de Arte no 9. Universidad de Málaga.

AMADOR DE LOS RIOS, Rodrigo (1907): Catálogo de los monumentos históricos y artísticos de la provincia de Málaga. Ejemplar mecanografiado de la Diputación Provincial de Málaga (Biblioteca Cánovas del Castillo).

Bejarano Robles, Francisco (1961): Catálogos de Documentos del reinado de los Reyes Católicos existentes en el Archivo Municipal de Málaga. Madrid.

Bejarano Robles, Francisco (1978): “Constitución del Concejo y del Primer Cabildo de Málaga." en Revista Jábega n²2. Centro de Ediciones de la Diputación de Málaga. Málaga.

Bejarano Robles, Francisco (1984): Las Calles de Málaga. De su historia y ambiente (Tomo I y II). Editorial Arguval. Málaga

BejARANO Robles, Francisco (1985): Los libros de Repartimiento de Málaga Tomo I. Ayuntamiento de Málaga. Málaga. 
Bejarano Robles, Francisco (1990): Los libros de Repartimiento de Málaga Tomo II. Ayuntamiento de Málaga. Málaga.

Bejarano Robles, Francisco (1998): Los libros de Repartimiento de Málaga Tomo III. Ayuntamiento de Málaga. Málaga 1998.

Burgos Madroñero, Manuel (1976): “Málaga, Ciudad Musulmana” en Revista Jábega no 15. Centro de Ediciones de la Diputación de Málaga. Málaga.

CAMACHO MARTÍNEZ, Rosario (1981): Málaga Barroca. Arquitectura religiosa de los siglos XVII y XVIII. Diputación, COA y Universidad de Málaga

CAMACHO MARTíNEZ, Rosario (1988): Arquitectura y Símbolo. Iconografía de la Catedral de Málaga. Málaga.

CAMACHO MARTíNEZ, Rosario (Dirección del equipo) (1985):.Inventario Artístico de Málaga y su provincia. Ministerio de Cultura. Madrid.

CAMACHO MARTíNEZ, Rosario (Dirección del equipo) (1997):.Guía históricoartística de Málaga. Málaga.

CAMACHO MARTíneZ, Rosario y ROMERO MARTíneZ, José María (1987): “La Iglesia del Sagrario de Málaga" en Asuntos de arquitectura nํㅜ 7. Colegio de Arquitectos de Málaga. Málaga.

CANDAU RAmILA, María Eugenia / DíAZ PARDO, José Ignacio / RODRIGUEZ MARÍN Francisco (2005) Guía de Arquitectura. Colegio Oficial de Arquitectos. Málaga.

Cerezo Moreno, José Luis y CARrera RodrígueZ, Francisco José. (1976): “La Iglesia de los Santos Mártires." en Revista Jábega nº15. Centro de Ediciones de la Diputación de Málaga. Málaga

CHueCA Goitia, Fernando. Historia de la Arquitectura Española. Edades Antiguas y Media. Editorial Dossat. Barcelona.

ChuecA Goitia, Fernando (1978): Breve historia del Urbanismo. Alianza Editorial, Madrid.

Del PINO, Enrique (2008): Historia general de Málaga. Editorial Almuzara. Córdoba.

DíAz DE EsCOBAR, José María (1932): Apuntes Históricos sobre algunas calles de Málaga. Estudios malagueños. Málaga.

GALÁN SÁNCHEZ, A. (1999): Málaga Musulmana. De la conquista a la guerra final. Siglos VIII al XV. Patrimonio Cultural de Málaga y su Provincia. 
GARCíA DE LA LeÑA, Cecilio. Conversaciones Históricas Malagueñas. Caja de Ahorros Provincial de Málaga. Málaga.

Guerrero-Strachan Carrillo, Jesús / Contreras López, Miguel Ángel (dirección y coordinación). (2009): Proyecto Trimalaga Departamento de Expresión Gráfica, Diseño y Proyectos de la ETSII. Universidad de Málaga.

GUILlÉN ROBLES, F. (1874): Historia de Málaga y su provincia. Málaga, reimpresión 1980, Ed. Arguval.

Guillen Robles, F. (1880): Málaga Musulmana. Editorial Arguval. Málaga.

JiMÉNEZ GUERRERO, José. (2011): La destrucción del patrimonio eclesiástico en la Guerra Civil. Málaga y su provincia. Editorial Arguval. Málaga.

LADERO QUESADA. M. A. (1983): Ejército, logística y finalización en la Guerra de Granada. Seis lecciones sobre la Guerra de Granada. Granada.

LADERO QUESADA. (1984): "Las ciudades de Andalucía occidental en la Baja Edad Media. Aspectos poblacionales y urbanísticos" Jornada de Historia Medieval. Junta de Andalucía, Jaen.

LAdero QuesadA. M. A. (1986): Coronas y ciudades en la Castilla del siglo XV. En la España Medieval V. Madrid.

LÓPEZ DE COCA, José Enrique (1977): La tierra de Málaga a finales del siglo XV. Universidad de Granada. Granada.

LÓPEZ DE COCA, José Enrique (1994): Málaga, del Islam al cristianismo (12391570). Historia de Málaga I. Málaga.

LÓPEZ SOLER, Mónica (2005): Iglesias de Málaga. Unicaja. Málaga.

LÓPEZ SOLER, Mónica (2011): Iglesias de Málaga. Editorial Almuzara, Jaén.

MachuCA SANTA-CruZ, Luis (1997): Málaga, Ciudad Abierta. Origen, cambio y permanencia de una estructura urbana. (2ª edición) Colegio Oficial de Arquitectos de Málaga. Málaga.

MOREJON P. (1999) Historia General y politica de los santos, antigüedades y grandezas de la ciudad de Málaga. Transcripción de Rafael Bejarano Pérez. Málaga.

OlANO GURRIARÁN, Cesar (1975): El desarrollo urbanístico de la ciudad de Málaga. Revista Jábega no 10 . Málaga.

PALENCIA, Alfonso de (1909). Guerra de Granada. Ed. Facsímil Universidad de Granada, 1998. 
RuIz Povedano, José María (2000): Málaga de Musulmana a Cristiana. La transformación de la ciudad a finales de la Edad Media. Ágora, Málaga.

SPENGLER, Oswald. (1946): La decadencia de Occidente. Bosquejo de una morfología de la Historia Universal. Vol. III, Espasa Calpe, Madrid.

Suberbiola MARTínEZ, Jesús (1975): “La Iglesia de Málaga, primera iglesia de Estado de España”. Jábega, no 10, 21-26.

YARZA LUACES, Joaquin / BORRAS GUALIS, Gonzalo (2000): Historia Universal del Arte. Bizancio e Islam, vol. IV, Espasa Calpe, Madrid. 